Article

\title{
Multivariate Multiscale Entropy Applied to Center of Pressure Signals Analysis: An Effect of Vibration Stimulation of Shoes
}

\author{
Qin Wei ${ }^{1}$, Dong-Hai Liu ${ }^{1}$, Kai-Hong Wang ${ }^{2}$, Quan Liu ${ }^{1}$, Maysam F. Abbod ${ }^{3}$, Bernard C. Jiang ${ }^{4}$, \\ Ku-Ping Chen ${ }^{5}$, Chuan $\mathrm{Wu}^{5}$ and Jiann-Shing Shieh ${ }^{2,6, *}$
}

1 School of Information Engineering, Wuhan University of Technology, Wuhan, 430070, China; E-Mails: zivaw2010@hotmail.com (Q.W.); liudhzhyym@163.com (D.H. L.); quanliu@whut.edu.cn (Q.L.)

2 Department of Mechanical Engineering, Yuan Ze University, Taoyuan, Chung-Li, 32003, Taiwan; E-Mail: s965038@mail.yzu.edu.tw (K.H.W.)

3 School of Engineering and Design, Brunel University, London, UB8 3PH, UK; E-Mail: maysam.abbod@brunel.ac.uk (M.F.A.)

4 Department of Industrial Engineering \& Management, Yuan Ze University, Taoyuan, Chung-Li, 32003, Taiwan; E-Mail: iebjiang@saturn.yzu.edu.tw (B.C.J.)

5 Taipei Veterans General Hospital, Taoyuan Branch, Taoyuan, 33052, Taiwan; E-Mails: nuredu@tyvh.gov.tw (K.P.C.);n107@tyvh.gov.tw (C.W.)

6 Center for Dynamical Biomarkers and Translational Medicine, National Central University, Chung-Li, 32001, Taiwan

* Author to whom correspondence should be addressed; E-Mail: jsshieh@saturn.yzu.edu.tw; Tel.: +886-3-463-8800 (ext. 2470); Fax: +886-3-455-8013.

Received: 7 September 2012; in revised form: 10 October 2012 / Accepted: 29 October 2012 / Published: 2 November 2012

\begin{abstract}
Falls are unpredictable accidents and resulting injuries can be serious to the elderly. A preventative solution can be the use of vibration stimulus of white noise to improve the sense of balance. In this work, a pair of vibration shoes were developed and controlled by a touch-type switch which can generate mechanical vibration noise to stimulate the patient's feet while wearing the shoes. In order to evaluate the balance stability and treatment effect of vibrating insoles in these shoes, multivariate multiscale entropy (MMSE) algorithm is applied to calculate the relative complexity index of reconstructed center of pressure (COP) signals in antero-posterior and medio-lateral directions by the multivariate empirical mode decomposition (MEMD). The results show that the balance stability of $61.5 \%$ elderly subjects is improved after wearing the developed shoes, which is
\end{abstract}


more than $30.8 \%$ using multiscale entropy. In conclusion, MEMD-enhanced MMSE is able to distinguish the smaller differences between before and after the use of vibration shoes in both two directions, which is more powerful than the empirical mode decomposition (EMD)-enhanced MSE in each individual direction.

Keywords: center of pressure; vibration; multivariate multiscale entropy; multivariate empirical mode decomposition

\section{Introduction}

Falls are a major public health concern among old-aged people, and they often cause serious injuries [1]. They most frequently occur during walking and are associated with the chronic deterioration in the neuromuscular and sensory systems, as well as with ankle muscle weakness and lower endurance of these muscles to fatigue [2]. Falls are a major cause of morbidity and mortality in the U.S., the medical research has shown that the degradation of physiological functions and nervous system functions makes the reduced the balance ability of the elderly, so they may easily fall down while walking [3]. In order to reduce the harm from falls in the elderly, it is suggested that the health care system develops a system which can improve and evaluate the sense of body balance. According to the phenomenon of stochastic resonance, which is based on the concept that the information transmitted through a system can be amplified by the presence of a particular level of noise [4], the noise is used to enhance the detection and transmission of weak signals in nonlinear system[5]. Furthermore, the particular mechanical noise is employed to improve the performance of the human balance control system through the eccentric rotating mass (ERM), linear resonant actuator (LRA), piezoelectric vibration generator (PVG) [6]. Besides, the stimulation from noise-based vibration devices such as vibrating insoles was employed to improve parkinsonian gait steadiness in a short-term setting [7].

The postural stability is an important feature to protect people from falls and to complete the desired actions, which can be measured by the displacement of center of pressure (COP) $[8,9]$. The COP displacements in the two anterior-posterior (AP) and medial-lateral (ML) directions are often used to characterize the COP stabilogram [10]. Furthermore, they are commonly applied to predict fall-risk [11] and evaluate balance stability enhancement systems [12]. Due to the non-stationary and non-linear characteristics of COP signals, our previous research demonstrated that the COP data analyzed by the multiscale entropy (MSE) is effectively better than that processed by the traditional method [13].

Nevertheless, MSE is suitable for one-dimensional time series, but not for the multivariate time series that are routinely measured in experimental and biological systems [14,15]. Based on empirical mode decomposition (EMD) [16] and MSE, their extensions such as multivariate EMD (MEMD) [17] and multivariate multiscale entropy (MMSE) $[18,19]$ were proposed to process the multichannel input for practical applications. Therefore, the multivariate time series from different channels can be decomposed by MEMD and analyzed by MMSE. According to the COP data containing ML and AP directions, the MEMD and MMSE adapt to processing the COP signals. Therefore, the aim of this paper is to measure the effectiveness of the MEMD-enhanced MMSE for analysis of COP signals in order to evaluate the balance stability when compared to the EMD-enhanced MSE presented in the 
previous study. Furthermore, it aims to verify the performance of the proposed vibration shoes through a comparison between before and after wearing the shoes. This paper is organized as follows: the analysis methods such as the MEMD and MMSE employed in this study are described in the next section. The third section introduces the developed vibration insoles, experimental subjects and experimental procedure. Then the results analyzed and compared by EMD-enhanced MSE and MEMD-enhanced MMSE are shown in the fourth section. Finally, we discuss the above results and draw the conclusions.

\section{Methods}

\subsection{Multivariate Empirical Mode Decomposition}

Since EMD was proposed by Huang et al. [16], it has been widely used for non-linear, non-stationary data analysis based on the intrinsic characteristics of time series. The intrinsic mode functions (IMFs) are decomposed by the mean $\mathrm{m}(\mathrm{t})$ of upper and down envelops of a time series $\mathrm{X}(\mathrm{t})$, expressed as follows:

$$
X(\mathrm{t})=\sum_{i=1}^{n} c_{i}+r_{n}
$$

where $c_{i}$ and $r_{n}$ are the $i$ th IMF and the last residue of time series. In order to expand the applications of EMD, MEMD, a multivariate extension of EMD, was presented to decompose multivariate nonlinear and nonstationary signals [20,21]. MEMD not only overcomes the single input limitation of EMD, but also solves the problem of mode mixing through addition of white noise to different channels. Furthermore, it is similar to EMD that acts as a dyadic filter bank on each channel of the multivariate input, and has the advantage of aligning the corresponding IMFs from different channels across the same frequency range [20]. Therefore, the more frequencies that exist in different channels, the more IMFs that are decomposed in each channel.

In computation of MEMD, the mean $\mathrm{m}(\mathrm{t})$ is calculated by means of the multivariate envelope curves of a set of $\mathrm{K}$ direction vectors, shown as equation (2):

$$
m(\mathrm{t})=K^{-1} \sum_{k=1}^{K} e^{\theta_{k}}(t)
$$

where $\left\{e^{\theta_{k}}(t)\right\}_{k=1}^{K}$ are the multivariate envelope curves of all vectors that are the projections of multichannel input $s(t)$ along $K$ directions. Then, the multivariate IMF is calculated by $s(t)-m(t)$ and the stoppage criterion. This process is repeated until the stoppage criteria in standard EMD is fulfilled by all the projected signals.

\subsection{Multivariate Multiscale Entropy}

In the entropy family, MSE is an effective method to evaluate the complexity of signals over different time scales, and has been applied effectively in the analysis of physiology, biology, and geosciences data [22-24]. There are two procedures in the MSE, the first one is coarse-grained procedure that computes the $y_{j}^{(\tau)}$ of original time series $\left\{\mathrm{x}_{1}, \mathrm{x}_{2}, \cdots, \mathrm{x}_{\mathrm{N}}\right\}$ based on the scale factor $\tau$, which is according to equation (3): 


$$
y_{j}^{(\tau)}=\frac{1}{\tau} \sum_{i=(j-1) \tau+1}^{j \tau} x_{i}
$$

Then the sample entropy (SampEn) [25] is employed to measure the degree of randomness of the finite length time series $\mathrm{y}_{j}^{(\tau)}$, in which $1 \leq j \leq N / \tau$. SampEn is considered as a conditional probability that is computed according to the following steps: firstly, two sequences of $m$ consecutive data points such as $Y_{m}(i)=\left\{y_{i}, \ldots, y_{i+m-1}\right\}$ and $Y_{m}(j)=\left\{y_{j}, \ldots, y_{j+m-1}\right\}$ are selected to compute the number of $Y_{m}(j)$ that meets the condition $d\left[Y_{m}(i), Y_{m}(j)\right] \leq \gamma * S . D .\left(j \in\left[1, N^{\prime}-m\right], i \neq j\right.$ and $\left.\gamma \in(0,1)\right)$, in which the S.D. is the standard deviation of the time series $\left\{\mathrm{y}_{1}, \mathrm{y}_{2}, \cdots, y_{\mathrm{N}^{\prime}}\right\}$ and $d\left[Y_{m}(i), Y_{m}(j)\right]$ is the maximum distance between $Y_{m}(i)$ and $Y_{m}(j)$ :

$$
d\left[Y_{m}(i), Y_{m}(j)\right]=\max \left[\left|y_{i+k}, y_{j+k}\right|\right](k \in[0, m-1])
$$

Then $B_{i}^{m}(\gamma)$ is the amount of all $Y_{m}(i)$ similar to $Y_{m}(j)$, and its average for $i \in\left[1, \mathrm{~N}^{\prime}-m\right]$ is:

$$
\mathrm{B}^{\mathrm{m}}(\gamma)=\left(\mathrm{N}^{\prime}-\mathrm{m}\right)^{-1} \sum_{\mathrm{i}=1}^{\mathrm{N}^{\prime}-\mathrm{m}} \mathrm{B}_{\mathrm{i}}^{\mathrm{m}}(\gamma)
$$

Thus, the $B^{m}(\gamma)$ is the average similarity of $m$ consecutive data points over whole $N^{\prime}-m$ data, and $B^{m+1}(\gamma)$ is that of $m+1$ consecutive data points. Finally, the SampEn is obtained using equation (6) :

$$
\operatorname{SampEn}\left(N^{\prime}, m, \gamma\right)=-\ln \left[\frac{B^{m+1}(\gamma)}{B^{m}(\gamma)}\right]
$$

Like MSE, the MMSE is used to calculate the relative complexity of normalized multichannel signals by plotting multivariate SampEn as a set of the scale factor. Its first step is to define temporal scales of the increased length by coarse-graining the $\mathrm{p}$-variate time series $\left\{\mathrm{x}_{\mathrm{k}, \mathrm{i}}\right\}_{\mathrm{i}=1}^{\mathrm{N}}, \mathrm{k}=1,2, \ldots, \mathrm{p}$. For a scale factor $\tau$, the multivariate coarse-grained time series $y_{k, j}^{(\tau)}$ are calculated as equation (7), where $1 \leq j \leq N / \tau$ :

$$
y_{k, j}^{(\tau)}=\frac{1}{\tau} \sum_{i=(j-1) \tau+1}^{j \tau} x_{k, i}
$$

But the second step is different from that in MSE, the multivariate SampEn (MSampEn) is calculated for each coarse-grained multivariate $y_{k, j}^{(\tau)}$, and it is plotted as a function of the scale factor $\tau$. In order to obtain the MSampEn, the multivariate emdedded vectors $Y_{m}(i) \in \mathbb{R}^{p}$ must be constructed firstly, which is shown as:

$$
\begin{array}{r}
Y_{m}(i)=\left[y_{1, i}, y_{1, i+\varepsilon_{1}}, \ldots, y_{1, i+\left(m_{1}-1\right) \varepsilon_{1}}, y_{2, i}, y_{2, i+\varepsilon_{2}}, \ldots,\right. \\
\left.y_{2, i+\left(m_{2}-1\right) \varepsilon_{2}}, \ldots, y_{p, i}, y_{p, i+\varepsilon_{p}}, \ldots, y_{p, i+}\left(m_{p}-1\right) \varepsilon_{p}\right]
\end{array}
$$

where $1 \leq i \leq \mathrm{N}^{\prime}-n$ and $\mathrm{N}^{\prime}=N / \tau, n=\max \{M\} \times \max \{\varepsilon\} . \quad M=\left[m_{1}, m_{2}, \ldots, m_{p}\right] \in \mathbb{R}^{p}$ is the embedding vector, while $\varepsilon=\left[\varepsilon_{1}, \varepsilon_{1}, \ldots, \varepsilon_{p}\right]$ is the time lag vector and $m=\sum_{k=1}^{p} m_{k}$. Then the maximum norm is defined by Chebysev distance between any two composite delay vectors $Y_{m}(i)$ and $Y_{m}(j)$, that is expressed as:

$$
d\left[Y_{m}(i), Y_{m}(j)\right]=\max _{l=1, \ldots, m}\{|\mathrm{y}(i+l-1)-\mathrm{y}(j+l-1)|\}
$$

where $j \in\left[1, \mathrm{~N}^{\prime}-n\right], j \neq i$. For a given $Y_{m}(i), P_{i}$ is the number of vector pairs that meets $d\left[Y_{m}(i), Y_{m}(j)\right] \leq \gamma * S . D . \quad\left(j \in\left[1, N^{\prime}-n\right], i \neq j\right.$ and $\left.\gamma \in(0,1)\right)$ where S.D. is the standard deviation of the 
multivariate emdedded vectors $\mathrm{Y}_{\mathrm{m}}(\mathrm{i})$, so that $A_{i}^{m}(\gamma)=P_{i} /\left(\mathrm{N}^{\prime}-n-1\right)$, where $n=\max \{M\} \times \max \{\tau\}$. For all $i, A^{m}(\gamma)=\left(\mathrm{N}^{\prime}-n\right)^{-1} \sum_{i=1}^{\mathrm{N}^{\prime}-n} A_{i}^{m}(\gamma)$.

Finally, the average similarity $A^{m}(\gamma)$ over all $i \in\left[1, \mathrm{~N}^{\prime}-n\right]$ and the $A^{m+1}(\gamma)$ over all $i \in[1, \mathrm{p} *$ $\left.\left(\mathrm{N}^{\prime}-n\right)\right]$ are used to gain the MSampEn, as shown in equation (10):

$$
\operatorname{MSampEn}\left(\mathrm{N}^{\prime}, M, \varepsilon, \gamma\right)=-\ln \left[\frac{A^{m+1}(\gamma)}{A^{m}(\gamma)}\right]
$$

where $\gamma$ is the tolerance level and $\mathrm{N}^{\prime}$ is the length of the time series $y_{k, j}^{(\tau)}$.

In MMSE, the multivariate time series are considered more complex than another if the MSampEn values are higher than other for majority of the scales, which is the same as the original MSE. The embedding vector $M=\left[m_{1}, m_{2}, \ldots, m_{p}\right] \in \mathbb{R}^{p}$ and the tolerance level $\gamma$ in MMSE have the equivalent function with parameters $m$ and $\gamma$ in MSE. In the following anaysis, $m$ is 2 and $\gamma$ is 0.2 in calculation of SampEn, and $M=\left[m_{1}, \ldots, m_{i}, \ldots, m_{p}\right], \varepsilon=\left[\varepsilon_{1}, \ldots, \varepsilon_{i}, \ldots, \varepsilon_{p}\right]\left(m_{i}=2, \varepsilon_{i}=1, i \in[1, p]\right)$, and $\gamma=0.2$ are set in MMSE so as to compare the performace between MSE and MMSE with the same parameters.

\section{Experiments}

\subsection{Experimental Devices}

The Danish Catsys 2000 system (DPD Company, Snekkersten, Denmark) is an advanced tool in quantitative analysis of some neurobehaviors such as tremors, postural stability, reaction time and coordination ability. It detects the movements in AP and ML directions as the COP recording of a subject, as shown in Figure 1. With a sampling rate of $31.25 \mathrm{~Hz}$ in this system, Figure 1 shows COP data of the subject collected in one minute. This device was used in our experiments to detect and record the COP data of all subjects.

Figure 1. An example of COP data recorded by the Catsys 2000.

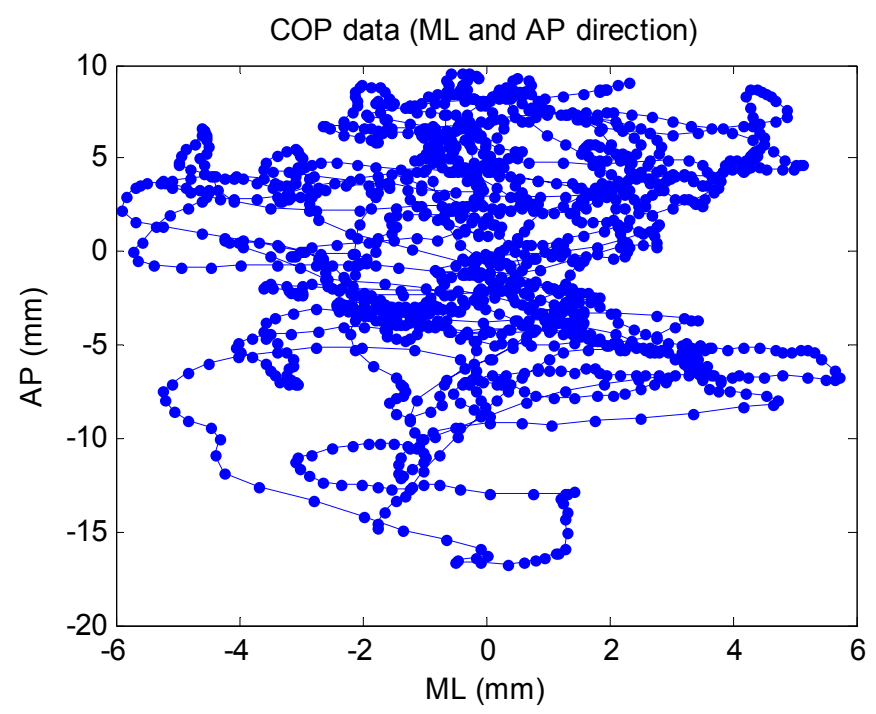

Moreover, we developed a pair of vibration shoes controlled by a touch-type switch which can generate mechanical vibration noises to stimulate the feet while patients are wearing these shoes. In these shoes, an insole with micro-motors is designed for the purpose of enhancing the stability of the 
elderly with presumed fall risk. Because of the drawbacks of ERM or LRA in response to the instantaneous signals and the PVG in generation of strong enough vibrations, therefore, the touch-type switch is preferred. According to the constant frequency vibrations which is controlled by the touch-type switch, if it is connected to the eccentric motor device in the insoles, the eccentric motor will start running when the feet press the touch-type switch. In our developed vibration shoes, each vibratory insole has three eccentric motors connected to three touch-type switches respectively, and there are two eccentric motors on the forefoot, and one on the heel. In addition, we design a variable resistor in vibratory insole which is controllable in accordance with the feelings of each subject to adjust the degree of vibration intensity.

\subsection{Experimental Subjects}

This study was approved by the Research Ethics Review Committee of the TaoYuan Veterans Hospital. After obtaining written informed consent from the subjects, we measured the COP data from 40 healthy subjects without any disease or fall history. They were divided into young group (21.95 \pm 2.16 years) and elderly group ( $58.7 \pm 4.77$ years). The feasibility of evaluation of balance stability was first verified by these obviously different two groups based on analysis of MSE and MMSE.

Secondly, in order to prove the effectiveness of the vibration shoes and the advantages of MMSE, the high-risk subjects (i.e., elderly group) who had a history of falling down more than twice in a year were chosen to measure the COP data before and after wearing our shoes. There were 26 elderly subjects $(84.1 \pm 4.1$ years $)$ in this group who had normal living habits without any disability.

\subsection{Experiment Procedure}

The experiment on the elderly subjects in the TaoYuan Veterans Hospital has three procedures. First, the subjects walk from their rooms to the experiment place one by one. Since the two places are in different buildings, the subjects were accompanied by a nurse and this would take at least 6-7 minutes. After the walk, the subjects stood on the non-invasive measurement physiological signals machine (i.e., Catsys 2000 system) to collect the COP data for one minute. Second, the subjects put on the vibratory shoes that supply the physical stimulation to the nerves on their feet. Subjects walk back and forth normally in an open place for six minutes. Third, the subjects are asked to stand on the non-invasive measurement physiological signals machine again for COP recording in one minute. Through these experimental procedures, we collected the COP data of all subjects during normal walking and after stimulation using the vibration shoes for the further analysis.

\section{Results}

\subsection{EMD-Enhanced MSE}

Analysis of COP data through EMD-enhanced MSE starts with EMD in order to decompose the COP data into IMFs, and then the appropriate IMFs are selected to reconstruct signals of AP and ML directions. For example, there are original signals of AP (COP-Y) and ML (COP-X) directions as shown in Figures 2(a) and (b), and the reconstructed IMF3+4 of the decomposed IMFs in each direction are displayed in Figures 2(c) and (d). The reconstructed signals of the two directions are 
smoother than the original signals at the cost of missing high frequency parts in IMF1 and IMF2. So far there is no clear frequency range in COP that is able to represent the balance stability of people. Thus, even though EMD has the dyadic filter bank property, different combinations of IMFs should be tested for further analysis of their complexity degree in statistics. The complexity degree of different combinations in each direction is measured by the complexity index (CI), which is defined as the area under the MSE curve over all scales [9]: $C I=\sum_{i=1}^{\text {scale }} \operatorname{SampEn}(i)$, in which scale is the maximum of the scale factors. The whole process of analyzing COP data based EMD-enhanced MSE is shown in Figure 3.

Figure 2. (a) Original COP data in ML direction. (b) Original COP data in AP direction. (c) Reconstructed COP data of IMF $3+4$ in ML direction. (d) Reconstructed COP data of IMF $3+4$ in AP direction.
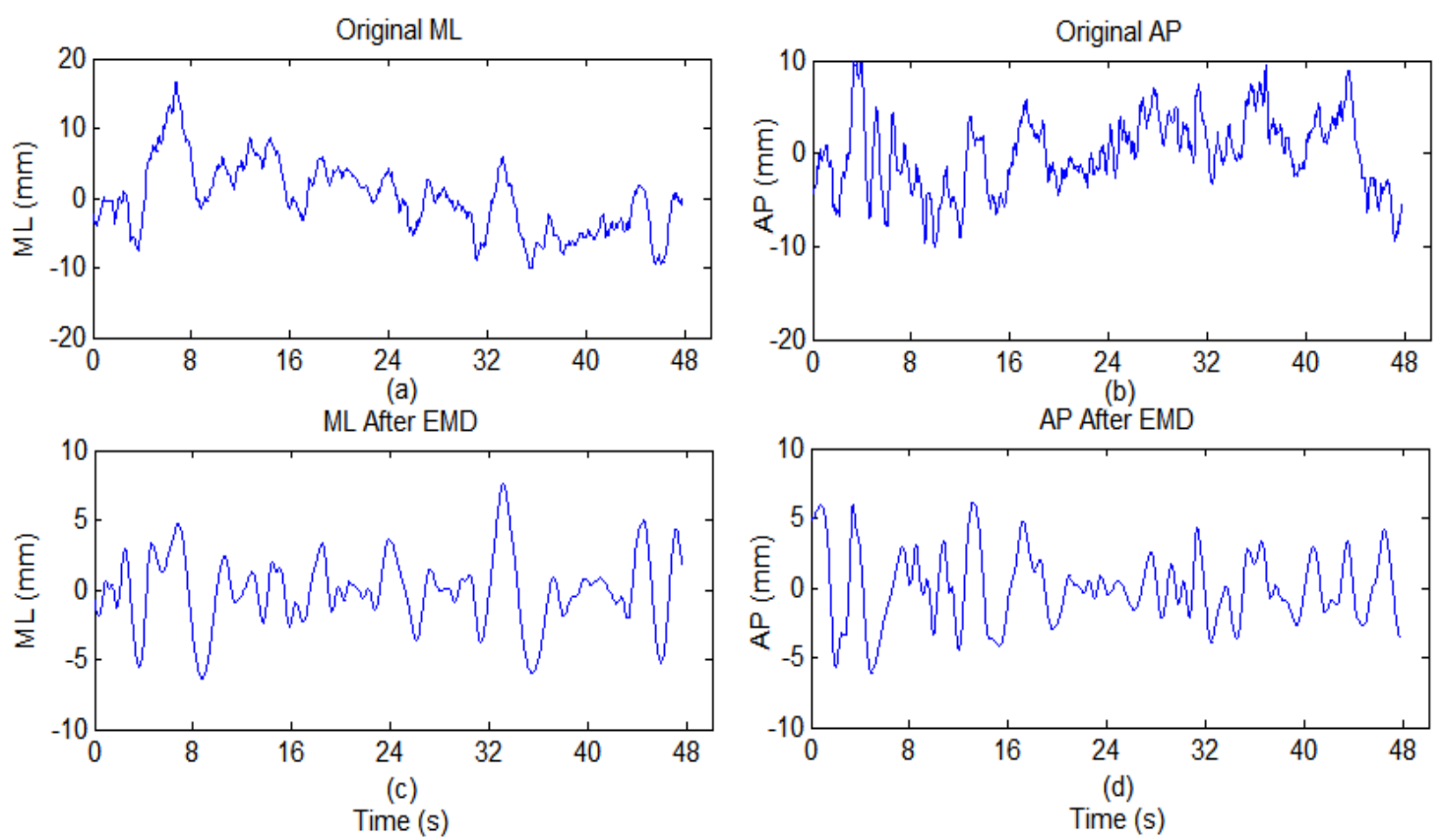

Figure 3. The procedure of computation of EMD-enhanced MSE.

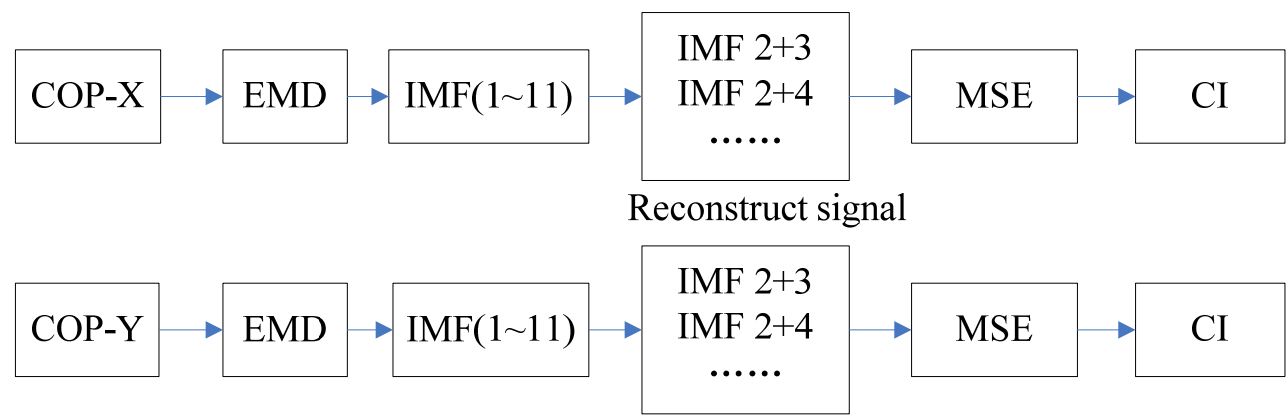

In the analysis of experimental results, there are 11 IMFs decomposed by original data through EMD. Which one or ones are appropriate to reconstruct the features of balance stability in signals of AP and ML directions? Because of the unclear meaning of each IMF, it is important to try the possible combinations from IMF 1 to IMF 11. After studying the frequency band of IMF 2-6, they are more suitable to reconstruct the signals of $\mathrm{AP}$ and $\mathrm{ML}$ directions due to the fact they contain more information from the original signals. Taking the CI in ML direction and AP direction, Table 1 shows 
the CI of the EMD-enhanced MSE values calculated by individual IMF or various combinations of IMFs. Reconstructed signals of IMF $3+4+5$, IMF $4+5$, and IMF 5 have statistically significant differences in complexity between the young and elderly subjects. Therefore these three combinations are selected to verify the effect of the vibration shoes further. Table 2 shows the CI of COP data and statistical results of paired t-test, but it is obvious that all three combinations of IMFs have no statistically significant difference $(p>0.05)$ before and after the use of vibration shoes.

Table 1. Mean and standard deviation of CI of EMD-enhanced MSE curve and independent $t$-test between young and elderly subjects for the twenty cases.

\begin{tabular}{|c|c|c|c|c|c|c|}
\hline \multirow{2}{*}{ IMF } & \multicolumn{3}{|c|}{ ML (x-direction) } & \multicolumn{3}{|c|}{ AP (y-direction) } \\
\hline & Young & Elderly & $p$-value & Young & Elderly & $p$-value \\
\hline 2 & $2.73 \pm 1.07$ & $2.74 \pm 1.30$ & 0.972 & $4.07 \pm 1.18$ & $2.92 \pm 1.03$ & 0.002 \\
\hline 3 & $3.05 \pm 0.94$ & $2.74 \pm 0.68$ & 0.240 & $3.44 \pm 0.72$ & $3.24 \pm 0.90$ & 0.444 \\
\hline 4 & $2.92 \pm 0.52$ & $2.36 \pm 0.49$ & 0.001 & $2.81 \pm 0.54$ & $2.56 \pm 0.61$ & 0.180 \\
\hline $5(*)$ & $1.95 \pm 0.48$ & $1.61 \pm 0.44$ & 0.026 & $2.00 \pm 0.49$ & $1.66 \pm 0.42$ & 0.024 \\
\hline 6 & $0.99 \pm 0.43$ & $0.84 \pm 0.27$ & 0.201 & $1.11 \pm 0.36$ & $0.79 \pm 0.30$ & 0.004 \\
\hline $2+3$ & $4.18 \pm 1.43$ & $3.93 \pm 1.30$ & 0.581 & $5.24 \pm 1.15$ & $3.93 \pm 1.30$ & 0.127 \\
\hline $2+4$ & $4.52 \pm 1.16$ & $4.07 \pm 1.14$ & 0.231 & $4.89 \pm 1.34$ & $4.48 \pm 1.40$ & 0.350 \\
\hline $2+5$ & $3.62 \pm 0.93$ & $3.73 \pm 1.47$ & 0.765 & $4.17 \pm 1.26$ & $3.80 \pm 1.12$ & 0.329 \\
\hline $2+6$ & $3.01 \pm 1.32$ & $3.54 \pm 1.58$ & 0.253 & $3.33 \pm 0.99$ & $3.22 \pm 1.33$ & 0.768 \\
\hline $3+4$ & $3.82 \pm 0.88$ & $3.16 \pm 0.51$ & 0.005 & $3.92 \pm 0.87$ & $3.50 \pm 0.83$ & 0.130 \\
\hline $3+5$ & $3.54 \pm 0.92$ & $3.04 \pm 0.74$ & 0.066 & $3.78 \pm 0.80$ & $3.26 \pm 0.64$ & 0.300 \\
\hline $3+6$ & $3.31 \pm 1.23$ & $2.85 \pm 0.68$ & 0.156 & $3.62 \pm 0.72$ & $3.02 \pm 0.86$ & 0.023 \\
\hline $4+5(*)$ & $2.58 \pm 0.45$ & $2.22 \pm 0.50$ & 0.019 & $2.71 \pm 0.41$ & $2.21 \pm 0.46$ & 0.001 \\
\hline $4+6$ & $2.42 \pm 0.69$ & $2.14 \pm 0.52$ & 0.156 & $2.45 \pm 0.52$ & $2.03 \pm 0.69$ & 0.034 \\
\hline $5+6$ & $1.54 \pm 0.63$ & $1.27 \pm 0.36$ & 0.105 & $1.68 \pm 0.39$ & $1.29 \pm 0.45$ & 0.005 \\
\hline $2+3+4$ & $4.73 \pm 1.12$ & $4.13 \pm 0.87$ & 0.066 & $5.12 \pm 1.33$ & $4.69 \pm 1.24$ & 0.301 \\
\hline $2+3+5$ & $4.37 \pm 1.15$ & $4.06 \pm 1.16$ & 0.402 & $4.91 \pm 1.11$ & $4.37 \pm 0.91$ & 0.106 \\
\hline $2+3+6$ & $4.20 \pm 1.51$ & $3.92 \pm 1.09$ & 0.507 & $4.71 \pm 0.98$ & $4.21 \pm 1.29$ & 0.177 \\
\hline $2+4+5$ & $3.66 \pm 0.83$ & $3.63 \pm 1.17$ & 0.931 & $4.17 \pm 1.11$ & $3.77 \pm 0.97$ & 0.244 \\
\hline $2+4+6$ & $3.67 \pm 1.20$ & $3.66 \pm 1.13$ & 0.977 & $3.70 \pm 0.92$ & $3.54 \pm 1.34$ & 0.652 \\
\hline $2+5+6$ & $2.91 \pm 1.05$ & $3.26 \pm 1.38$ & 0.369 & $3.21 \pm 0.83$ & $3.05 \pm 1.21$ & 0.615 \\
\hline $3+4+5(*)$ & $3.39 \pm 0.66$ & $2.96 \pm 0.60$ & 0.037 & $3.67 \pm 0.70$ & $3.19 \pm 0.62$ & 0.026 \\
\hline $3+4+6$ & $3.38 \pm 0.89$ & $2.92 \pm 0.57$ & 0.057 & $3.51 \pm 0.68$ & $3.06 \pm 0.81$ & 0.060 \\
\hline $3+5+6$ & $3.00 \pm 0.90$ & $2.73 \pm 0.67$ & 0.286 & $3.41 \pm 0.72$ & $2.82 \pm 0.82$ & 0.021 \\
\hline $4+5+6$ & $2.29 \pm 0.61$ & $2.07 \pm 0.59$ & 0.249 & $2.49 \pm 0.37$ & $1.99 \pm 0.58$ & 0.003 \\
\hline $2+3+4+5$ & $4.09 \pm 0.84$ & $3.94 \pm 0.98$ & 0.604 & $4.69 \pm 1.12$ & $4.25 \pm 0.88$ & 0.175 \\
\hline $2+3+4+6$ & $4.17 \pm 1.15$ & $3.90 \pm 0.95$ & 0.425 & $4.41 \pm 0.98$ & $4.05 \pm 1.24$ & 0.319 \\
\hline $2+3+5+6$ & $3.79 \pm 1.17$ & $3.68 \pm 1.10$ & 0.758 & $4.33 \pm 0.93$ & $3.85 \pm 1.16$ & 0.157 \\
\hline $2+4+5+6$ & $3.24 \pm 0.94$ & $3.39 \pm 1.13$ & 0.648 & $3.66 \pm 0.82$ & $3.67 \pm 1.12$ & 0.352 \\
\hline $3+4+5+6$ & $3.12 \pm 0.71$ & $2.77 \pm 0.62$ & 0.110 & $3.41 \pm 0.56$ & $2.90 \pm 0.73$ & 0.020 \\
\hline $2+3+4+5+6$ & $3.77 \pm 0.90$ & $3.71 \pm 0.98$ & 0.842 & $4.23 \pm 0.93$ & $3.89 \pm 1.07$ & 0.282 \\
\hline
\end{tabular}

Note: The combinations of IMFs are marked with asterisk when $p<0.05$ in both ML and AP directions. 
Table 2. Mean and standard deviation of CI of EMD-enhanced MSE curve and paired $t$-test between before and after the use of vibration shoes.

\begin{tabular}{ccccccc}
\hline \multirow{2}{*}{ IMF } & \multicolumn{3}{c}{ ML (x-direction) } & \multicolumn{3}{c}{ AP (y-direction) } \\
\cline { 2 - 7 } & Before & After & $\boldsymbol{p}$-value & Before & After & $\boldsymbol{p}$-value \\
\hline $\mathbf{5}$ & $1.52 \pm 0.33$ & $1.56 \pm 0.44$ & 0.702 & $1.87 \pm 0.51$ & $1.90 \pm 0.36$ & 0.803 \\
$\mathbf{4 + 5}$ & $2.26 \pm 0.41$ & $2.18 \pm 0.51$ & 0.439 & $2.51 \pm 0.53$ & $2.58 \pm 0.52$ & 0.641 \\
$\mathbf{3 + 4 + 5}$ & $3.03 \pm 0.40$ & $2.98 \pm 0.52$ & 0.731 & $3.56 \pm 0.85$ & $3.64 \pm 0.82$ & 0.733 \\
\hline
\end{tabular}

\subsection{MEMD-Enhanced MMSE}

As shown in Figure 4, the COP data including signals of AP and ML directions and two added white noise signals are the multivariate input for the MEMD. According to the flat power spectral density of white noise and the property of dyadic filter bank in MEMD, the two added white noise signals not only are helpful to solve the mixing mode, but also decompose the IMFs with more detailed frequency ranges. Then the output of MEMD is a matrix of IMFs, which includes the IMFs of AP (COP-Y), ML(COP-X) and two white noise signals, furthermore, each input signal has the same number of decomposed IMFs. After reconstruction using different combinations in each direction of AP and ML, the same combination of IMFs in individual direction becomes the multi-input of the MMSE for the integrated complexity of balance stability. For example, the combination of IMF $2+4$ in AP direction is chosen as one input for MMSE, so that the IMF2+4 in ML direction should be the other input for consistency and integrated complexity.

Figure 4. The procedure of computation of MEMD-enhanced MMSE.

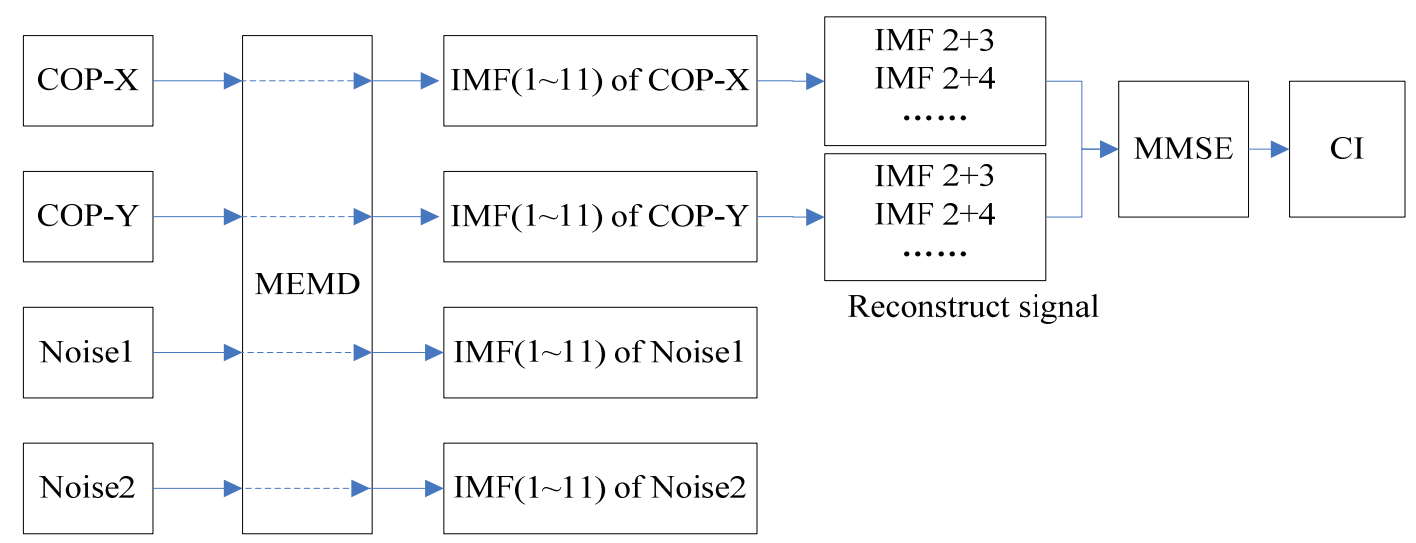

Compared to three in the EMD-enhanced MSE, there are 16 combinations that have the significant differences (i.e., $p<0.05$ ) between the young and elderly subjects through MEMD-enhanced MMSE, shown in Table 3. The four combinations with $p<0.005$ in Table 3 are asterisk-marked. Furthermore, it is apparent that the MEMD-enhanced MMSE has better performance in analysis of the differences before and after wearing the vibration shoes and the improvement of balance stability. In Table 4 , the mean of the CI calculated by the combinations such as IMF 4 , IMF $2+4$, IMF $3+4$ and IMF $2+3+4$ has a relatively higher value after putting on our shoes to improve the sense of balance. The CI of these four combinations has a statistically significant difference $(p<0.05)$ before and after the use of 
vibration shoes. Therefore, it is proven that the $\mathrm{CI}$ is effective in measuring the balance stability through MEMD-enhanced MMSE in comparison with EMD-enhanced MSE.

Table 3. CI of MEMD-enhanced MMSE curve and p-value of young group and elderly group (Independent-Samples T Test).

\begin{tabular}{|c|c|c|c|}
\hline IMF & Young & Elderly & $p$-value \\
\hline 2 & $4.07 \pm 0.57$ & $3.74 \pm 0.68$ & 0.117 \\
\hline 3 & $4.18 \pm 0.74$ & $3.69 \pm 1.04$ & 0.095 \\
\hline $4(*)$ & $4.57 \pm 0.85$ & $3.61 \pm 1.00$ & 0.002 \\
\hline 5 & $4.23 \pm 0.73$ & $3.70 \pm 0.94$ & 0.053 \\
\hline 6 & $4.10 \pm 0.77$ & $3.83 \pm 0.64$ & 0.237 \\
\hline $2+3$ & $4.82 \pm 0.77$ & $4.31 \pm 1.16$ & 0.114 \\
\hline $2+4(*)$ & $5.18 \pm 1.03$ & $4.01 \pm 1.18$ & 0.002 \\
\hline $2+5(*)$ & $4.63 \pm 0.75$ & $3.91 \pm 1.08$ & 0.019 \\
\hline $2+6$ & $4.30 \pm 0.84$ & $3.92 \pm 0.61$ & 0.111 \\
\hline $3+4(*)$ & $5.26 \pm 0.96$ & $4.18 \pm 1.04$ & 0.002 \\
\hline $3+5(*)$ & $5.13 \pm 0.69$ & $4.24 \pm 1.16$ & 0.006 \\
\hline $3+6$ & $4.77 \pm 0.89$ & $4.28 \pm 0.62$ & 0.051 \\
\hline $4+5(*)$ & $5.08 \pm 0.83$ & $4.31 \pm 1.06$ & 0.015 \\
\hline $4+6(*)$ & $5.13 \pm 0.78$ & $4.59 \pm 0.70$ & 0.026 \\
\hline $5+6$ & $4.26 \pm 0.65$ & $4.34 \pm 0.62$ & 0.687 \\
\hline $2+3+4(*)$ & $5.59 \pm 1.10$ & $4.47 \pm 1.18$ & 0.003 \\
\hline $2+3+5(*)$ & $5.41 \pm 0.71$ & $4.41 \pm 1.24$ & 0.004 \\
\hline $2+3+6(*)$ & $4.92 \pm 0.91$ & $4.36 \pm 0.61$ & 0.029 \\
\hline $2+4+5\left(^{*}\right)$ & $5.31 \pm 0.93$ & $4.42 \pm 1.12$ & 0.010 \\
\hline $2+4+6(*)$ & $5.28 \pm 0.84$ & $4.66 \pm 0.69$ & 0.015 \\
\hline $2+5+6$ & $4.38 \pm 0.68$ & $4.41 \pm 0.64$ & 0.864 \\
\hline $3+4+5(*)$ & $5.54 \pm 0.89$ & $4.61 \pm 1.15$ & 0.007 \\
\hline $3+4+6(*)$ & $5.51 \pm 0.87$ & $4.84 \pm 0.75$ & 0.014 \\
\hline $3+5+6$ & $4.60 \pm 0.75$ & $4.55 \pm 0.68$ & 0.828 \\
\hline $4+5+6$ & $4.88 \pm 0.68$ & $4.74 \pm 0.77$ & 0.552 \\
\hline $2+3+4+5\left(^{*}\right)$ & $5.75 \pm 0.97$ & $4.74 \pm 1.24$ & 0.007 \\
\hline $2+3+4+6(*)$ & $5.61 \pm 0.89$ & $4.94 \pm 0.74$ & 0.013 \\
\hline $2+3+5+6$ & $4.62 \pm 0.78$ & $4.53 \pm 0.68$ & 0.707 \\
\hline $2+4+5+6$ & $4.97 \pm 0.71$ & $4.79 \pm 0.80$ & 0.457 \\
\hline $3+4+5+6$ & $5.15 \pm 0.76$ & $4.89 \pm 0.82$ & 0.308 \\
\hline $2+3+4+5+6$ & $5.28 \pm 0.81$ & $4.95 \pm 0.84$ & 0.227 \\
\hline
\end{tabular}

Note: The combinations of IMFs are marked with asterisk while $p$ value $<0.05$.

Table 4. CI of MEMD-enhanced MMSE curve and $p$-value before and after the use of vibration shoes (Paired-Samples T Test).

\begin{tabular}{cccc}
\hline IMF & Before & After & p-value \\
\hline $\mathbf{4}$ & $3.06 \pm 1.13$ & $3.74 \pm 0.96$ & 0.028 \\
$\mathbf{2 + 4}$ & $3.55 \pm 1.35$ & $4.32 \pm 1.18$ & 0.037 \\
$\mathbf{3 + 4}$ & $3.78 \pm 1.38$ & $4.64 \pm 1.21$ & 0.027 \\
$\mathbf{2 + 3 + 4}$ & $4.03 \pm 1.47$ & $4.93 \pm 1.30$ & 0.030 \\
\hline
\end{tabular}




\subsection{Comparison between EMD-Enhanced MSE and MEMD-Enhanced MMSE in Analysis of COP}

When comparing COP of young and elderly subjects, there are three combinations with $p<0.05$ in both AP and ML directions through the EMD-enhanced MSE as shown in Table 1, however, sixteen combinations with $p<0.05$ are obtained using the MEMD-enhanced MMSE. Besides, the variation of entropic parameters with the scale factors is also different for the two methods. For instance, the combinations of IMF2+4 in both cases are selected to reconstruct signals of AP and ML directions. In Figure 5, the SampEn and MSampEn curves are shown over scales of 1 to 7, in which the young group is marked as blue line/circle symbols and the elderly group is red line/star symbols. Due to the fact that $\mathrm{AP}$ and ML are independent directions, MSE only can process each of them separately as shown in Figures 5(a,b), however, Figure 5(c) shows the MMSE curves in two directions together. It is obvious that the blue circles are higher than red stars over the all scales in Figure 5(c), which fully complies with the common phenomenon that the young group usually has better sense of balance than the elderly group. On the other hand, in Figures 5(a) and (b), the SampEn of young subjects are close to that of elderly subjects with scales of 1, 6 and 7, while the distance of SampEn between young and elderly subjects are apparently smaller than that of MSampEn for each scale. Moreover, in the example of the combination of IMF2+4, the statistical analyses of CI in both AP and ML directions based on EMD-enhanced MSE shows no significant differences between young and elderly groups as shown in Table 1. Nevertheless, it has significant difference $(p<0.05)$ based on the MEMD-enhanced MMSE as shown in Table 3. It means that MEMD-enhanced MMSE is more sensitive in detecting the differences between young and elderly subjects. Therefore, the CI with combinations in EMD-enhanced MSE has statistically significant differences between young and elderly group but does not show that before and after using the vibration shoes, as shown in the Tables 1 and 2. On the contrary, there is statistically significant CI difference which is not only between young and elderly group but also between before and after using the vibration shoes through MEMD-enhanced MMSE, as displayed in Tables 3 and 4.

Finally, in order to observe the individual differences of improvement in sense of balance in details, results of 26 elderly subjects who have falling records are shown in Table 5. The CI and its difference between before and after the use of vibration shoes in each case are compared between the two methods on the basis of the combination of IMF $2+3+4$. In these 26 cases, there are 16 cases with increasing degree of complexity in COP data through MEMD-enhanced MMSE, but only eight cases, half of the former, appear in the analysis of EMD-enhanced MSE. Therefore, based on the combination of IMF $2+3+4$, the MEMD-enhanced MMSE has the results of $61.5 \%$ elderly subjects with improved balance stability in comparison with 30.8\% in EMD-enhanced MSE.

According to the results shown in Tables 1 and 3, even though the EMD-enhanced MSE and MEMD-enhanced MMSE are both feasible to analyze and measure the balance stability, they have different performance in the amount of appropriate combinations and the sensitivity for detecting the distinct complexity of balance stability between young and elderly subjects. These results indicate that the MEMD-enhanced MMSE is more sensitive in detecting differences in the balance stability between young and elderly subjects. Moreover, the MSE and MMSE curves of the combination of IMF $2+4$ as shown in Figure 5 indicates that MSampEn is better than SampEn in analysis of multiple signals and have bigger difference over the whole scale factors. Under this condition, the results shown in Table 5 not only indicate that MEMD-enhanced MMSE has better performance in analysis of COP, but also 
prove that the vibration insoles controlled by touch-type switch can effectively improve the sense of balance for elderly people.

Figure 5. Mean and standard deviation between young and elderly groups analyzed by MSE and MMSE for the twenty cases. (a) MSE in ML direction. (b) MSE in AP direction. (c) MMSE in both AP and ML directions.

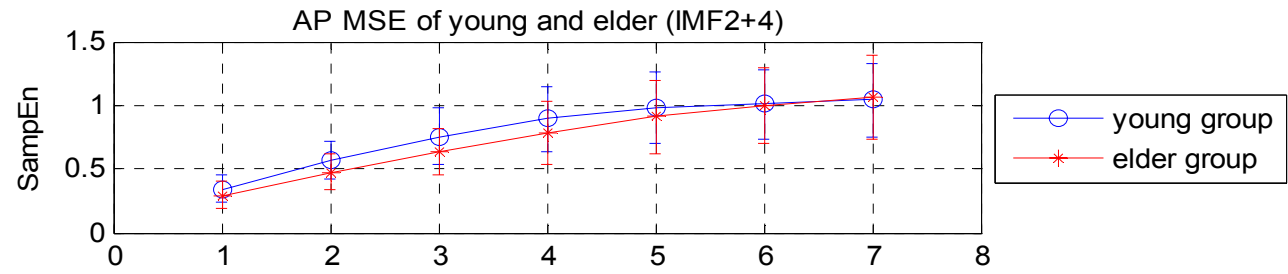

(a)

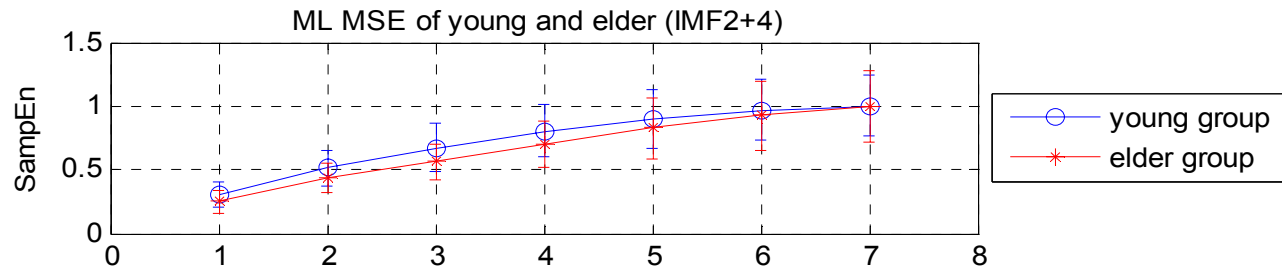

(b)

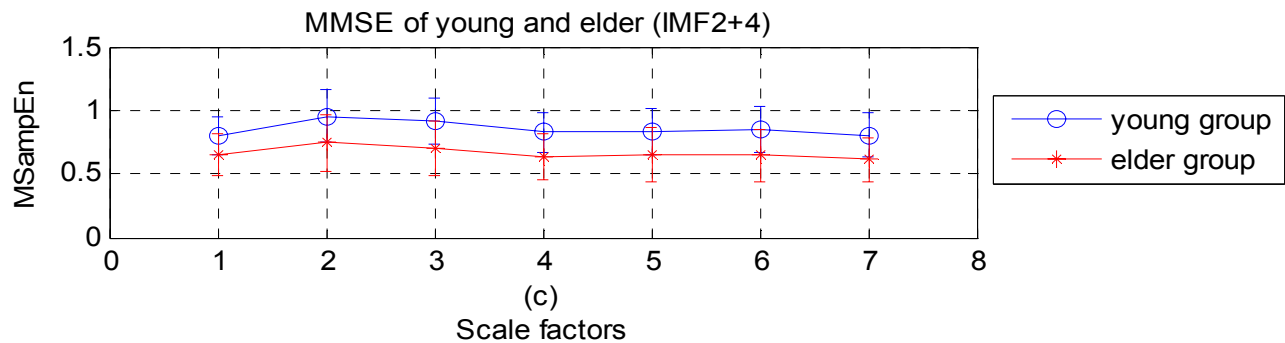

Table 5. CI of EMD-enhanced MSE and MEMD-enhanced MMSE curve and p-value in Paired-Samples $t$-test between before and after the use of vibration shoes. Diff $=(\mathrm{CI}$ after vibration $)$ - (CI before vibration).

\begin{tabular}{c|c|c|c|c|c|c|c|c|c}
\hline \multirow{2}{*}{ ID } & \multicolumn{3}{|c|}{ MMSE } & \multicolumn{5}{c}{ MSE } \\
\cline { 2 - 11 } & \multicolumn{3}{|c|}{ ML\&AP } & \multicolumn{3}{c}{ ML (x-axis) } & \multicolumn{3}{c}{ AP (y-axis) } \\
\cline { 2 - 11 } & Before & After & Diff & Before & After & Diff & Before & After & Diff \\
\hline $\mathbf{1}$ & 3.50 & 4.51 & 1.01 & 3.45 & 4.59 & 1.13 & 3.63 & 6.99 & 3.36 \\
$\mathbf{2}$ & 3.76 & 3.54 & -0.22 & 4.39 & 4.48 & 0.08 & 6.29 & 5.46 & -0.83 \\
$\mathbf{3}$ & 3.52 & 4.75 & 1.23 & 3.51 & 4.16 & 0.65 & 5.18 & 5.30 & 0.12 \\
$\mathbf{4}$ & 3.07 & 4.84 & 1.77 & 3.42 & 3.06 & -0.35 & 5.76 & 4.77 & -1.00 \\
$\mathbf{5}$ & 5.57 & 4.66 & -0.91 & 2.75 & 4.97 & 2.22 & 5.15 & 5.95 & 0.80 \\
$\mathbf{6}$ & 2.95 & 5.49 & 2.55 & 3.69 & 2.08 & -1.61 & 3.51 & 6.35 & 2.84 \\
$\mathbf{7}$ & 4.42 & 5.51 & 1.09 & 5.30 & 3.87 & -1.44 & 6.48 & 5.16 & -1.32 \\
$\mathbf{8}$ & 2.11 & 5.43 & 3.33 & 4.13 & 4.64 & 0.51 & 6.60 & 6.57 & -0.02 \\
$\mathbf{9}$ & 5.70 & 5.51 & -0.19 & 4.37 & 5.75 & 1.38 & 6.10 & 6.54 & 0.43 \\
$\mathbf{1 0}$ & 2.62 & 1.88 & -0.75 & 3.94 & 4.32 & 0.38 & 5.24 & 4.26 & -0.97 \\
$\mathbf{1 1}$ & 1.05 & 6.14 & 5.10 & 3.07 & 4.51 & 1.44 & 1.37 & 6.38 & 5.02 \\
$\mathbf{1 2}$ & 2.68 & 5.97 & 3.29 & 3.35 & 3.61 & 0.26 & 5.23 & 4.67 & -0.57 \\
$\mathbf{1 3}$ & 4.34 & 4.25 & -0.09 & 4.14 & 2.58 & -1.57 & 5.97 & 6.00 & 0.03 \\
\hline
\end{tabular}


Table 5. Cont.

\begin{tabular}{|c|c|c|c|c|c|c|c|c|c|}
\hline \multirow{3}{*}{ ID } & \multirow{2}{*}{\multicolumn{3}{|c|}{$\begin{array}{c}\text { MMSE } \\
\text { ML\&AP }\end{array}$}} & \multicolumn{6}{|c|}{ MSE } \\
\hline & & & & \multicolumn{3}{|c|}{ ML (x-axis) } & \multicolumn{3}{|c|}{ AP (y-axis) } \\
\hline & Before & After & Diff & Before & After & Diff & Before & After & Diff \\
\hline 14 & 5.51 & 5.70 & 0.19 & 5.75 & 4.37 & -1.38 & 6.54 & 6.10 & -0.43 \\
\hline 15 & 3.02 & 5.97 & 2.95 & 3.16 & 4.83 & 1.67 & 4.01 & 6.48 & 2.47 \\
\hline 16 & 3.98 & 3.50 & -0.48 & 4.86 & 3.71 & -1.15 & 5.50 & 5.26 & -0.24 \\
\hline 17 & 5.21 & 5.37 & 0.16 & 3.45 & 3.68 & 0.22 & 5.58 & 5.30 & -0.28 \\
\hline 18 & 5.68 & 0.96 & -4.71 & 6.94 & 4.16 & -2.78 & 7.00 & 0.56 & -6.44 \\
\hline 19 & 5.82 & 5.50 & -0.32 & 3.11 & 3.43 & 0.33 & 5.92 & 4.40 & -1.53 \\
\hline 20 & 2.14 & 5.35 & 3.20 & 3.66 & 3.78 & 0.12 & 3.50 & 4.79 & 1.29 \\
\hline 21 & 5.67 & 5.40 & -0.27 & 4.63 & 3.35 & -1.28 & 7.41 & 6.39 & -1.02 \\
\hline 22 & 6.24 & 5.48 & -0.76 & 4.08 & 4.12 & 0.03 & 4.92 & 4.10 & -0.82 \\
\hline 23 & 6.42 & 6.64 & 0.22 & 5.81 & 6.34 & 0.53 & 6.91 & 8.17 & 1.25 \\
\hline 24 & 3.76 & 4.49 & 0.73 & 3.51 & 3.45 & -0.06 & 4.80 & 4.38 & -0.43 \\
\hline 25 & 3.02 & 6.55 & 3.53 & 4.01 & 3.33 & -0.69 & 5.55 & 5.38 & -0.16 \\
\hline 26 & 2.86 & 4.74 & 1.88 & 3.84 & 3.85 & 0.02 & 6.31 & 5.34 & -0.96 \\
\hline Mean \pm SD & $\begin{array}{c}4.03 \pm \\
1.47\end{array}$ & $\begin{array}{c}4.93 \pm \\
1.30\end{array}$ & $\begin{array}{c}0.91 \pm \\
2.00\end{array}$ & $\begin{array}{c}4.09 \pm \\
0.98\end{array}$ & $\begin{array}{c}4.04 \pm \\
0.90\end{array}$ & $\begin{array}{c}-0.05 \pm \\
1.18\end{array}$ & $\begin{array}{c}5.40 \pm \\
1.34\end{array}$ & $\begin{array}{c}5.43 \pm \\
1.38\end{array}$ & $\begin{array}{c}0.02 \pm \\
2.06\end{array}$ \\
\hline$p$-value & \multicolumn{3}{|c|}{0.03} & \multicolumn{3}{|c|}{0.827} & \multicolumn{3}{|c|}{0.955} \\
\hline Improve & \multicolumn{3}{|c|}{$16 / 26=61.5 \%$} & \multicolumn{6}{|c|}{$8 / 26=30.8 \%$} \\
\hline
\end{tabular}

\section{Discussion}

Although MEMD-enhanced MMSE is shown to be very promising technique in this study, there are still some combinations of IMFs with relevant CI as shown in Table 3. These kinds of combinations in Tables 1 and 3 represent that the IMFs involved have no features of balance stability or only have parts of them which are impossible to measure by MSE and MMSE. Therefore, the MEMD-enhanced MMSE still has several problems when using this combination method. Firstly, it is difficult to determine which IMF includes the valuable physiological features in these bio-signals. In our previous studies of the Monte Carlo verification [26,27], it can distinguish the properties of IMFs between noise and bio-signals. However, it is still impossible to recognize which IMFs are dominant factors for these diseases or events accurately. Although the obvious physiological response of patients within the particular frequency band through Valsalva maneuver test [28] is helpful to trace the IMF that is more related to this event, it is still intended to test for this kind of experiment. Most bio-signal analysis situations do not have this kind of patient stimulation. Therefore, the physiological features of bio-signals related to diseases or events are the key of choosing the IMFs that are more important. Currently, the lack of theoretical support in neuroscience for controlling balance of human body has led to the use of trial and error feedback to decide the IMFs which are dominant factors in this study. Additionally, there are even 16 combinations with the significant difference between young and elderly groups in Table 3, but some combinations cannot discriminate the small improvement achieved by the vibration shoes. It may be related to the meaning of each IMF, thus, these combination of IMFs need to be validated in future research. 
Secondly, there is a biasing problem when increasing the scales in MSE, and it is not well adapted to the nonlinear and nonstationary signals within a low sampling rate through the coarse-graining procedure. Recently, an adaptive multiscale entropy (AME) [29] and the MSE based on adaptive sampling procedure [30] were presented to solve this problem. For MMSE, however, the research on the influence of variation of parameters and its computation performance is our focus in the next stage, while the problem caused by coarse-graining procedure in MMSE will be studied in the future works.

Finally, the calculation in MEMD-enhanced MMSE is time consuming and memory-intensive. It is suggested that a very large internal memory is necessary if it is calculated on a desktop style computer. Also, there are several tricks in Matlab for more efficient computation, for instance, if there is a multiple-core processor in your computer, you can specify that in the preamble of Matlab and then computation will be more efficient. Moreover, the general-purpose computing on the graphics processing unit (GPGPU) is also able to enhance the performance of computation, which has already been applied in calculating the ensemble empirical-mode decomposition (EEMD) to improve its efficiency [31]. Therefore, a desktop style computer with more powerful and large internal memory can solve these problems in the computation of MEMD-enhanced MMSE in the near future.

\section{Conclusions}

To conclude, the developed vibration shoes are able to improve the sense of balance in elderly subjects through the noise stimulation. Though the EMD-enhanced MSE and MEMD-enhanced MMSE are able to distinguish the differences between young and elderly subjects, the latter are more sensitive to the changes between before and after the use of vibration shoes in both two directions, which is more powerful than the former in each individual direction.

\section{Acknowledgments}

The authors wish to thank the Exchange Student Program of Yuan Ze University in Taiwan and Wuhan University of Technology in China for supporting this research. This research was financially supported by the National Science Council (NSC) of Taiwan (NSC 99-2221-E-155-046-MY3). This research was also supported by the Center for Dynamical Biomarkers and Translational Medicine, National Central University, Taiwan which is sponsored by National Science Council (NSC100-2911-I-008-001). Furthermore, it was supported by the National Science Foundation of China (No.50935005).

\section{References}

1. Roudsari, B.S.; Ebel, B.E.; Corso, P.S.; Molinari, N.M.; Koepsell, T.D. The acute medical care costs of fall-related injuries among the U.S older adults. J. Care Injured. 2005, 36, 1316-1322.

2. Gefen, A. Simulations of foot stability during gait characteristic of ankle dorsiflexor weakness in the elderly. IEEE Trans. Neur. Sys. Reh. 2001, 9, 333-337.

3. Jiang, S.S.; Zhang, B.F.; Wei, D.M. The elderly fall risk assessment and prediction based on gait analysis. In Proceedings of 2011 IEEE 11th International Conference on Computer and Information Technology (CIT), Pafos, Cyprus, 31 August-2 September 2011; pp. 176-180. 
4. McDonnell, M.D.; Stocks, N.G.; Pearce, C.E.M.; Derek A. Stochastic Resonance: from Supra Threshold Stochastic Resonance to Stochastic Signal Quantization, 1st ed.; Cambridge University Press: Cambridge, UK, 2008; pp. 6-20.

5. Moss, F.; Ward, L.M.; Sannita, W.G. Stochastic resonance and sensory information processing: a tutorial and review of application. Clin. Neurophysiol. 2004, 115, 267-281.

6. Liu, W.; Lipsitz, L.A.; Montero-Odasso, M.; Bean, J.; Kerrigan, D.C.; Collins, J.J. Noise-enhanced vibrotactile sensitivity in older adults, patients with stroke, and patients with diabetic neuropathy. Arch. Phys. Med. Rehabil. 2002, 83,171-176.

7. Novak, P.; Novak, V. Effect of step-synchronized vibration stimulation of soles on gait in Parkinson's disease: a pilot study. J. Neuroeng. Rehabil. 2006, 3, 9-15.

8. Pajala, S.; Era, P.; Koskenvuo, M.; Kaprio, J.; Törmäkangas, T.; Rantanen, T. Force platform balance measures as predictors of indoor and outdoor falls in community-dwelling women aged 63-76 years. J. Gerontol. A 2008, 63,171-178.

9. Costa. M.; Priplata, A.A.; Lipsitz, L.A.; Wu, Z.; Huang, N.E.; Goldberger, A.L.; and Peng, C.-K. Noise and poise: Enhancement of postural complexity in the elderly with a stochastic-resonance-based therapy. Europhys. Lett. 2007, 68008.

10. Chen, S.J.; Gielo-Perczak, K. Effect of impeded medial longitudinal arch drop on vertical ground reaction force and center of pressure during static loading. Foot Ankle Int. 2011, 32, 77-84.

11. Van Wegan, E.E.H.; Van Emmerik, R.E.A.; Riccio, G.E. Postural orientation: Age-related changes in variability and time-to-boundary. Hum. Movement Sci. 2002, 21, 61-68.

12. Lai, C.-L.; Tseng S.-Y.; Huang, C.-H.; Pei, C.; Chi, W.-M.; Hsu, L.-C.; Sun, T.-L. Fun and accurate static balance training to enhance fall prevention ability of aged adults: A preliminary study. Hum. Factors Ergonom. Manuf. Serv. Ind. 2012, doi:10.1002/hfm.20345.

13. Jiang, B.C.; Yang, W.-H.; Shieh, J.-S.; Fan, S.-Z.; Peng, C.-K. Entropy-based method for COP data analysis. Theor. Issues Ergonomics Sci. 2012, doi: 10.1080/1463922X.2011.617109.

14. Costa, M.; Goldberger, A.L.; Peng, C.-K. Multiscale entropy analysis of biological signals. Phys. Rev. E. 2005, doi: 10.1103/PhysRevE.71.021906.

15. Sarkar, A.; Barat, P., Multiscale entropy analysis: A new method to detect determinism in a time series. Entropy 2006, 8, 1-9.

16. Huang, N.E.; Shen, Z.; Long, S.R.; Wu, M.C.; Shih, H.H.; Zheng, Q.; Yen, N.C.; Tung, C.C.; Liu, H.H. The empirical mode decomposition and Hilbert spectrum for nonlinear and nonstationary time series analysis. Proc. R. Soc. Lond. A 1998, 454, 903-995.

17. Rehman, N.; Mandic, D.P. Multivariate empirical mode decomposition. Proc. R. Soc. A 2010, 466, 1291-1302.

18. Ahmed, M.U.; Mandic, D.P. Multivariate multiscale entropy: A tool for complexity analysis of multichannel data. Phys. Review E. 2011, 061918.

19. Morabito, F.C.; Labate, D.; La Foresta, F.; Bramanti, A.; Morabito, G.; Palamara, I., Multivariate Multi-Scale Permutation Entropy for Complexity Analysis of Alzheimer's Disease EEG. Entropy 2012, 14, 1186-1202.

20. Rehman, N.; Mandic, D.P. Filter bank property of multivariate empirical mode decomposition. IEEE Trans. Signal Proc. 2010, 59, 2421-2426. 
21. Zhou, X.; Yang, T.; Zou, H.-L.; Zhao, H. Multivariate empirical mode decomposition approach for adaptive denoising of fringe patterns. Opt. Lett. 2012, 37, 1904-1906.

22. Costa, M.; Goldberger, A.L.; Peng, C.K. Multiscale entropy analysis of complex physiologic time series. Phys. Rev. Lett. 2002, doi: 10.1103/PhysRevLett.89.068102.

23. Costa, M.; Peng, C.-K.; Goldberger, A.L.; Hausdorff, J.M. Multiscale entropy analysis of human gait dynamics. Physica A 2003, 330, 53-60.

24. Thuraisingham, R.A.; Gottwald, G.A. On multiscale entropy analysis for physiological data. Physica A 2006, 366, 323-332.

25. Richman, J.; Moorman, J. Physiological time series analysis using approximate entropy and sample entropy. Am. J. Physiol. 2000, 278, H2039-H2049.

26. Yeh, J.R.; Shieh, J.S.; Huang, N.E. Complementary ensemble empirical mode decomposition: A novel noise enhanced data analysis method. Adv. Adapt. Data Anal. 2010, 02, 135-156.

27. Yeh, J.R.; Lin, T.-Y.; Chen, Y.; Sun, W.-Z.; Abbod, M.F.; Shieh, J.-S. Investigating properties of the cardiovascular system using innovative analysis algorithms based on ensemble empirical mode decomposition. Comp. Math. Method Med. 2012, $2012,943431$.

28. Hu, K.; Peng C.K.; Czosnyka, M.; Zhao, P.; Novak, V. Nonlinear assessment of cerebral autoregulation from spontaneous blood pressure and cerebral blood flow fluctuations. Cardiovasc. Eng. 2008, 8, 60-71.

29. Hu, M; Liang, H.L. Adaptive multiscale entropy analysis of multivariate neural data. IEEE Trans. Biomed. Eng. 2012, 59, 12-15.

30. Liu, Q.; Wei, Q.; Fan, S.-Z.; Lu, C.-W.; Lin, T.-Y.; Abbod, M.F.; Shieh, J.-S. Adaptive computation of multiscale entropy and its application in EEG signals for monitoring depth of anesthesia during surgery. Entropy 2012, 14, 978-992.

31. Chen, D.; Li, D.; Xiong, M.Z.; Bao, H.; Li, X.L. GPGPU-aided ensemble empirical-mode decomposition for EEG analysis during anesthesia. IEEE Trans. Inf. Technol. B. 2010, 14, $1417-1427$.

(C) 2012 by the authors; licensee MDPI, Basel, Switzerland. This article is an open access article distributed under the terms and conditions of the Creative Commons Attribution license (http://creativecommons.org/licenses/by/3.0/). 